OPEN ACCESS

Edited by:

Zhiwei Wu,

Nanjing Medical University, China

Reviewed by:

Davide Angeletti,

University of Gothenburg, Sweden

Tongqing Zhou,

National Institutes of Health $(\mathrm{NIH})$,

United States

*Correspondence:

Daniela Fera

dfera1@swarthmore.edu

Specialty section:

This article was submitted to

Viral Immunology,

a section of the journal

Frontiers in Immunology

Received: 11 February 2020

Accepted: 10 June 2020

Published: 17 July 2020

Citation:

Zhou JO, Zaidi HA, Ton T and Fera D (2020) The Effects of Framework Mutations at the Variable Domain Interface on Antibody Affinity Maturation in an HIV-1 Broadly Neutralizing Antibody Lineage.

Front. Immunol. 11:1529. doi: 10.3389/fimmu.2020.01529

\section{The Effects of Framework Mutations at the Variable Domain Interface on Antibody Affinity Maturation in an HIV-1 Broadly Neutralizing Antibody Lineage}

\author{
Jeffrey O. Zhou ${ }^{1}$, Hussain A. Zaidi ${ }^{1}$, Therese Ton ${ }^{2}$ and Daniela Fera ${ }^{1 *}$ \\ ${ }^{1}$ Department of Chemistry and Biochemistry, Swarthmore College, Swarthmore, PA, United States, ${ }^{2}$ Department of Biology, \\ Swarthmore College, Swarthmore, PA, United States
}

Understanding affinity maturation of antibodies that can target many variants of HIV-1 is important for vaccine development. While the antigen-binding site of antibodies is known to mutate throughout the co-evolution of antibodies and viruses in infected individuals, the roles of the mutations in the antibody framework region are not well understood. Throughout affinity maturation, the $\mathrm{CH} 103$ broadly neutralizing antibody lineage, from an individual designated $\mathrm{CH} 505$, altered the orientation of one of its antibody variable domains. The change in orientation was a response to insertions in the variable loop 5 (V5) of the HIV envelope. In this study, we generated $\mathrm{CH} 103$ lineage antibody variants in which residues in the variable domain interface were mutated, and measured the binding to both autologous and heterologous HIV-1 envelopes. Our data show that very few mutations in an early intermediate antibody of the lineage can improve binding toward both autologous and heterologous HIV-1 envelopes. We also crystallized an antibody mutant to show that framework mutations alone can result in a shift in relative orientations of the variable domains. Taken together, our results demonstrate the functional importance of residues located outside the antigen-binding site in affinity maturation.

Keywords: human immunodeficiency virus (HIV), antibody, evolution, crystal structure, somatic hypermutation, framework

\section{INTRODUCTION}

A well-recognized aspect of antibody affinity maturation is the somatic hypermutation (SHM) of the antigen binding regions, i.e., the complementarity determining regions, or CDRs (1). This is even observed in antibodies that target rapidly evolving pathogens, such as HIV-1 and influenza. Antibodies target the viral spike, the envelope (Env) or hemagglutinin (HA), in the case of HIV-1 and flu, respectively $(2,3)$. In certain cases, antibodies that can neutralize a broad spectrum $(>50 \%)$ of viral variants can be achieved and these antibodies typically target certain conserved regions of the viral spike. In the case of HIV-1, such broadly neutralizing antibodies (bnAbs) arise in $\sim 10-20 \%$ of chronically infected patients after about 5 years of infection. The long time frame is partly due to the fact that the virus rapidly develops escape mutations to avoid antibody recognition $(4,5)$. This results in bnAbs with high mutation frequencies. 
Several bnAb lineages have been identified that target HIV1 Env or flu HA and they each use different strategies during the affinity maturation process to alter their CDRs to bind the viral spike. In the CAP256-VRC26 bnAb lineage, a disulfide bond was introduced within the heavy chain CDR loop 3 (HCDR3) that rigidified and properly oriented it for binding HIV-1 Env, thereby leading to breadth $(6,7)$. Rigidification of the HCDR3 was also important in the development of breadth of the CH65CH67 lineage against flu HA (8). In other cases, structural changes of the paratope were not observed. Instead, there were mutations within the CDRs that introduced residues or rotamers of residues necessary for interacting with Env. This was observed in the DH270 and $\mathrm{CH} 235$ antibody lineages where improbable mutations were necessary in interacting with key components of Env and/or Env glycans (9-12). Deletions and/or insertions in the CDRs could also alter the angle of approach of antibodies with Env, allowing them to accommodate glycans on Env, as observed in the PGT121 bnAb lineage (13-16).

While CDRs are responsible for the majority of the direct contacts made with an antigen, SHM does also occur in antibodies outside of their CDRs, in the intervening framework regions (FWRs). While the FWRs help stabilize the antigenbinding site and define the conformations of the CDR loops, the roles of mutations in the FWR are not well-understood. Reports have shown that FWR mutations can be responsible for the thermal destabilization of HIV-1 bnAbs, but not weakly neutralizing HIV-1 antibodies $(17,18)$. FWR mutations have also been shown to increase the dynamics of antibodies, leading to neutralization breadth $(17,19,20)$.

The $\mathrm{CH} 103$ bnAb lineage, derived from patient $\mathrm{CH} 505$, produced several bnAbs including $\mathrm{CH} 103$, which target the CD4 receptor binding site (CD4bs) of the HIV-1 Env (21). Crystal structures of antibody fragments from this lineage showed that the orientation of the heavy chain variable domain $\left(\mathrm{V}_{\mathrm{H}}\right)$ changed relative to the light chain variable domain $\left(\mathrm{V}_{\mathrm{L}}\right)$ in the transition from intermediate antibody I3.2 to I2 during affinity maturation, potentially through FWR mutations (22). While the CD4bs is relatively conserved, rapid mutations in the nearby Env variable loop 5 (V5) can lead to resistance against CD4bs antibodies (23). In the $\mathrm{CH} 505$ patient, Env insertion mutations in V5 occurred throughout virus evolution, which reduced the potency of $\mathrm{CH} 103$ lineage bnAb precursors through steric interference (22). In response to these insertions, over the course of affinity maturation the antibody $\mathrm{V}_{\mathrm{L}}$ domain shifted away from $\mathrm{V}_{\mathrm{H}}$ and the Env V5 loop to accommodate the V5 loop insertions. Different $\mathrm{V}_{\mathrm{L}}$ orientations relative to $\mathrm{V}_{\mathrm{H}}$ were observed in the structures of intermediate antibody I3.2 and of the chimeric antibody, I3.1. These antibodies both contain the $\mathrm{V}_{\mathrm{H}} \mathrm{DJ}_{\mathrm{H}}$ of I3, but this is paired with either the $\mathrm{V}_{\mathrm{L}} \mathrm{J}_{\mathrm{L}}$ of the unmutated common ancestor (UCA) in I3.2 or with the $\mathrm{V}_{\mathrm{L}} \mathrm{J}_{\mathrm{L}}$ of the more mature intermediate antibody I2 in I3.1. Thus, this revealed that the shift in $\mathrm{V}_{\mathrm{L}}$ was mainly attributed by the identity of the antibody's light chain.

To determine if FWR mutations, specifically ones in the antibody light chain at the $\mathrm{V}_{\mathrm{H}}-\mathrm{V}_{\mathrm{L}}$ interface, were responsible for the shift observed in the $\mathrm{CH} 103$ lineage and breadth development, we performed binding measurements to show that these mutations improved affinity toward an autologous (strain specific) HIV-1 Env and heterologous (different strain) HIV-1 Env with longer V5 loops. We also determined the crystal structure of a mutant, which shows that two residues in the FWR at the $\mathrm{V}_{\mathrm{H}}-\mathrm{V}_{\mathrm{L}}$ interface could lead to a large part of the observed shift seen in the $\mathrm{V}_{\mathrm{L}} \mathrm{J}_{\mathrm{L}}$ of the UCA and I2 between intermediate antibodies I3.2 and I2. Thermal melts showed that stability was not altered. Our analysis suggests that few mutations can have a large impact on antigen binding, and that antigen distal FWR mutations occurring during antibody maturation can lead to the structural properties necessary to improve affinity toward an antigen without altering stability.

\section{RESULTS}

\section{Antibody Interface Mutations Improve Binding to Autologous and Heterologous HIV-1 Envs With Longer V5 Loops}

During infection of the $\mathrm{CH} 505$ donor, insertion mutations were introduced in the V5 loop of the $\mathrm{CH} 505 \mathrm{HIV}-1$ envelopes as they evolved (21). While the UCA and early intermediate antibodies had a structure whose $\mathrm{V}_{\mathrm{L}}$ domain, specifically the antibody DE loop residues $65_{\mathrm{L}}-67_{\mathrm{L}}$, would clash with V5 loops that were longer than that of the $\mathrm{CH} 505$ transmitted founder $(\mathrm{T} / \mathrm{F})$ Env, the relative shift in $\mathrm{V}_{\mathrm{L}}$ with respect to $\mathrm{V}_{\mathrm{H}}$ appeared to accommodate these V5 loop insertions as well as the longer V5 loops found in heterologous HIV-1 Envs (Figure 1A). As a result, autologous $\mathrm{CH} 505$ Envs with longer V5 loops displayed differential binding to different members of the $\mathrm{CH} 103$ antibody lineage (i.e., they bound better to later members of the lineage than earlier members), whereas the CH505 T/F Env bound with similar affinity to all CH103 lineage members (Table 1) (22).

To assess the role of residues at the $\mathrm{V}_{\mathrm{H}}-\mathrm{V}_{\mathrm{L}}$ interface on binding Env, we generated an Fab mutant, "I32M", which consists of two interface mutations in the light chain of I3.2 (i.e., the UCA $V_{L} J_{L}$ ). The light chain mutations we introduced, $Q 38_{L} V$ and $L 46_{L} \mathrm{~V}$, were based on residues found in the mature members of the lineage (Figures 1B,C). To test binding to HIV-1 Env, we performed biolayer interferometry (BLI) with gp120 core, the monomeric form of the HIV-1 Env ectodomain. Because we wanted to identify differential binding capabilities, we tested binding to an autologous CH505 gp120 T/F core with a threeresidue "ETF" V5 loop insertion, which occurred during the natural evolution of the virus in the $\mathrm{CH} 505$ patient. I32M bound significantly better than I3.2 to this CH505 gp120 core and with a similar $K_{D}$ as the chimeric antibody I3.1, which was previously shown to have a $V_{L}$ shifted relative to $V_{H}$ (22) (Figure 2A, Table 1), demonstrating the importance to binding of the two mutations at the $\mathrm{V}_{\mathrm{H}}-\mathrm{V}_{\mathrm{L}}$ interface, $\mathrm{Q} 38_{\mathrm{L}} \mathrm{V}$ and $\mathrm{L} 46_{\mathrm{L}} \mathrm{V}$. The CH505 T/F wild-type gp120 core did not bind differentially to $\mathrm{CH} 103$ lineage members, as expected, nor to our mutant, I32M, which had similar $K_{D}$ (Table 1). These results suggest that the mutations we introduced did not disrupt binding to Env and that they improve binding to autologous Envs with significantly longer V5 loops. 

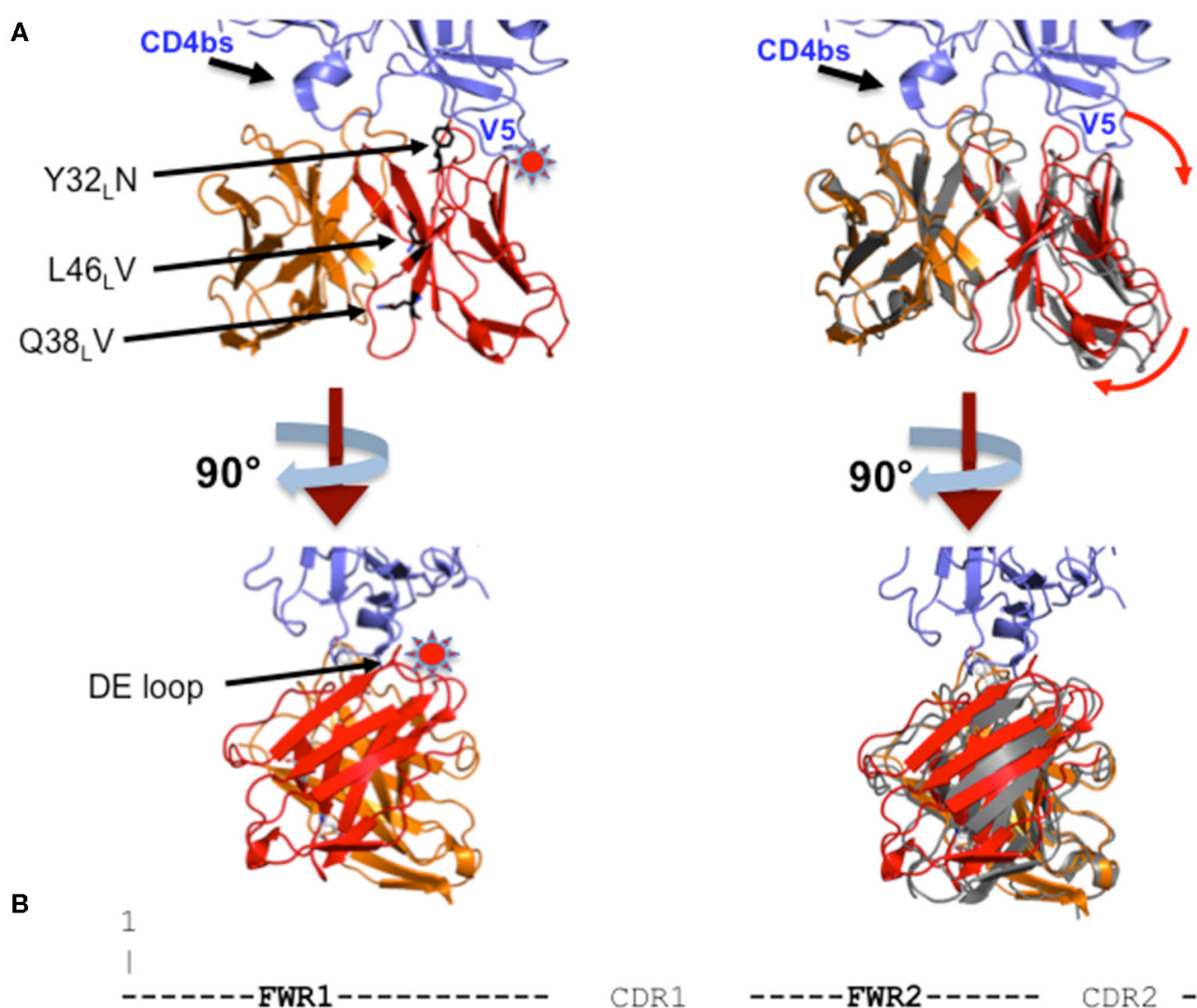

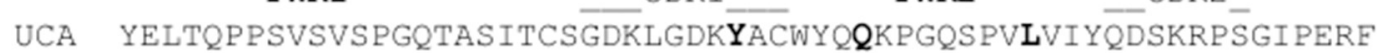

I 32M YELTQPPSVSVSPGQTASITCSGDKLGDKYACWYQVKPGQSPVVVIYQDSKRPSGI PERF

I 33M YELTQPPSVSVSPGQTAS ITCSGDKLGDKNACWYQVKPGQSPVVVIYQDSKRPSGI PERE

I2 YELTQPPSVSVSPGQTATITCSGDKVASKNVCWYQVKPGQSPEVVMYENYKRPSGIPDRF

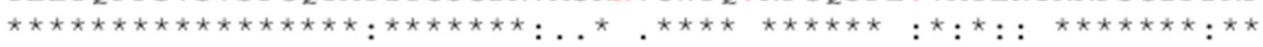

61

106

|

------FWR3------------ CDR3 ---FWR4---

UCA SGSNSGNTATLTISGTQAMDEADYYCQAWDSESTFVEGTGTKVTV

I32M SGSNSGNTATLTISGTQAMDEADYYCQAWDSFSTFVFGTGTKVTV

I 33M SGSNSGNTATLTISGTQAMDEADYYCQAWDSFSTFVEGTGTKVTV

I2 SGSKSGSTATLTIRGTQATDEADYYCQVWDSESTFVFGSGTQVTV

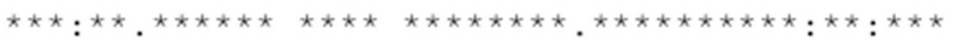

C

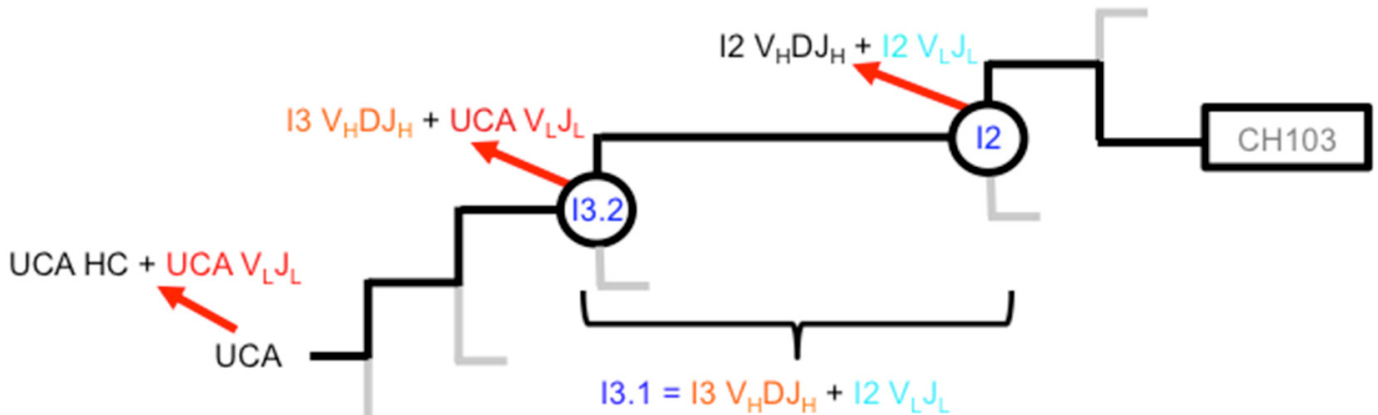

FIGURE 1 | Comparison of wild-type and mutant Fabs. (A) Superposition of the I3.2 Fab with the CH103/gp120 outer domain complex. Two orthogonal views of the variable region of the I3.2 Fab (heavy chain $\mathrm{V}_{\mathrm{H}}$ in orange, light chain $\mathrm{V}_{\mathrm{L}}$ in red) superposed onto the variable region of the $\mathrm{CH} 103 \mathrm{Fab}$ (gray) in complex with the 
FIGURE 1 | gp120 outer domain (blue) from ZM176.66 [Protein Data Bank (PDB) ID code 4JAN]. The V5 and CD4 binding site loops of gp120 are marked. A star indicates location of steric clash. Interface mutations introduced in this study are shown as black sticks. Image created in PyMOL. (B) Sequence alignment of the variable light chains of $\mathrm{I} 3$ wild-type and mutant Fabs. Mutations analyzed in this study are highlighted in red. $\left(^{*}\right)$ fully conserved residue, (:) strong conservation of properties, (.) weak conservation of properties, a blank space indicates little to no conservation of properties. (C) $\mathrm{CH} 103$ bnAb clonal lineage tree. The part of the $\mathrm{CH} 103$ lineage under study is shown. Gray lines represent other branches in the lineage. The UCA is shown on the left, the intermediates of interest are circled, and the $\mathrm{CH} 103$ bnAb has a rectangle around it. Heavy and light chain compositions are indicated with red arrows. The chimeric antibody is also defined based on its heavy and light chain compositions.

TABLE 1 | Dissociation constants $\left(K_{D}\right.$, in $\left.\mu \mathrm{M}\right)$ between $\mathrm{CH} 103$ lineage Fabs and gp120 core variants.

\begin{tabular}{|c|c|c|c|c|}
\hline & $\begin{array}{c}\text { CH505 T/F } \\
\text { gp120 core } \\
\text { WT }\end{array}$ & $\begin{array}{l}\text { CH505 T/F } \\
\text { gp120 core } \\
\text { V5 ETF } \\
\text { mutant* }^{\star}\end{array}$ & $\begin{array}{l}\text { 92UG037.8 } \\
\text { gp120 core } \\
\text { WT }\end{array}$ & $\begin{array}{c}\text { 92UG037.8 } \\
\text { gp120 core V5 } \\
\text { mutant** }^{\star}\end{array}$ \\
\hline 13.2 & $1.06 \pm 0.09$ & $>100$ & $>100$ & - \\
\hline I32M & $0.98 \pm 0.06$ & $15.51 \pm 4.11$ & $41.89 \pm 8.26$ & - \\
\hline I33M & $1.86 \pm 0.11$ & $3.70 \pm 0.18$ & $16.17 \pm 2.21$ & - \\
\hline |3.1 & $1.98 \pm 0.34$ & $23.74 \pm 2.77$ & $3.28 \pm 0.83$ & $10.5 \pm 0.5^{\star \star \star}$ \\
\hline UCA WT & $2.40 \pm 0.04^{\star \star \star}$ & NB & NB & $52.23 \pm 6.37$ \\
\hline UCA Q39 ${ }_{H} \mathrm{~L}$ & - & - & - & $25.10 \pm 2.43$ \\
\hline UCA S56HE & - & - & - & $6.98 \pm 0.44$ \\
\hline $\begin{array}{l}\text { UCA Q39 } 9_{H} L \text {, } \\
\text { S56 } 6_{H} E\end{array}$ & - & - & - & $2.20 \pm 0.30$ \\
\hline
\end{tabular}

"A three residue "ETF" insertion was added to the V5 loop of wild-type CH505 T/F gp120 core.

**V5 loop residues "GNINES" of wild-type 92UG037.8 gp120 core were replaced with "KNNT" of CH505 T/F virus Env.

${ }^{\star \star \star}$ Published elsewhere (22).

NB denotes no binding detected.

Dashes indicate combinations not tested.

A hallmark of breadth is the ability of antibodies to bind and neutralize heterologous virus strains. To determine if our I32M mutant could also display improved binding toward heterologous Envs, we tested binding to HIV-1 strain 92UG037.8, whose sequence in the V5 loop differs and is two amino acids longer than that of the $\mathrm{CH} 505 \mathrm{~T} / \mathrm{F}$ Env. Analysis by BLI shows that I32M binds to this Env with a significantly smaller $K_{D}$ than its wild-type counterpart I3.2, but not as well as the chimeric antibody I3.1 (Figure 2B, Table 1). These results suggest that two mutations alone in the $\mathrm{V}_{\mathrm{H}}-\mathrm{V}_{\mathrm{L}}$ interface improve binding to both autologous and heterologous Envs with longer V5 loops, and thereby contribute to breadth against different HIV-1 strains, however, additional light chain mutations may further improve binding to heterologous Envs.

\section{Few Mutations in the UCA Can Result in Heterologous HIV-1 Env Binding}

The UCA is the ancestor of an antibody lineage that is produced against the autologous virus that causes infection. In the UCA of the $\mathrm{CH} 103$ lineage, $\mathrm{Q} 39_{\mathrm{H}}$ in the heavy chain forms a reciprocal side-chain hydrogen bond with the light chain $\mathrm{Q} 38_{\mathrm{L}}$ at the $\mathrm{V}_{\mathrm{H}^{-}}$ $\mathrm{V}_{\mathrm{L}}$ interface (22). Moreover, the I3.2 early intermediate antibody has 18 mutations, only in its heavy chain, when compared to the UCA (Supplementary Figure 1). To determine whether mutations at the $\mathrm{V}_{\mathrm{H}}-\mathrm{V}_{\mathrm{L}}$ interface can improve binding to the progenitor antibody, we introduced the $\mathrm{Q} 39_{\mathrm{H}} \mathrm{L}$ mutation in its heavy chain and tested binding by BLI. Since the UCA of the $\mathrm{CH} 103$ lineage binds with a similar affinity to the CH505 $\mathrm{T} / \mathrm{F}$ gp120 core as other members of the lineage and it cannot bind to $\mathrm{CH} 505$ or heterologous gp120 cores with longer V5 loops (Table 1), we tested a 92UG037.8 gp120 core whose V5 loop was replaced with a shorter one from the $\mathrm{CH} 505 \mathrm{~T} / \mathrm{F}$ Env (22). While binding is fairly weak, comparison to the binding of the wild-type UCA shows that the introduction of this single mutation can improve binding by a factor of two (Figure 2C, Table 1). To ensure that the rapid association and dissociation rates observed were not due to bulk shifts at such high gp120 core concentrations, we also tested binding to the VRC01 bnAb, which recognizes the CD4bs but with a different approach angle $(24,25)$. This bnAb displayed slower on and off rates, distinguishing its binding kinetics from those of $\mathrm{CH} 103$ lineage members and their corresponding mutants (Supplementary Table 1, Supplementary Figure 2). This result suggests that the disruption of the $\mathrm{V}_{\mathrm{H}}-\mathrm{V}_{\mathrm{L}}$ interface $\mathrm{Q} 39_{\mathrm{H}}-\mathrm{Q} 38_{\mathrm{L}}$ reciprocal side-chain hydrogen bond is important in affinity maturation and can even improve binding of the earliest antibody lineage member.

Because binding to the UCA Q39 ${ }_{\mathrm{H}} \mathrm{L}$ mutant was still relatively weak $\left(K_{D}=25.10 \mu \mathrm{M}\right)$, we wanted to see if any mutations could further significantly improve binding to the heterologous Env. The published CH103 bnAb-gp120 structure shows that E56 $6_{\mathrm{H}}$ of the $\mathrm{CH} 103$ bnAb heavy chain HCDR2 loop engages in hydrogen bonding contacts with the backbone of the conserved gp120 CD4bs (21). Thus, we introduced the S56 $6_{\mathrm{H}} \mathrm{E}$ mutation into the UCA Q39 $\mathrm{H}$ L mutant and tested binding to the 92UG037.8 gp120 core V5 mutant. The UCA Q39 $9_{\mathrm{H}} \mathrm{L}, \mathrm{S} 56_{\mathrm{H}} \mathrm{E}$ double mutant could bind to the 92UG037.8 gp120 core V5 mutant with low micromolar affinity $\left(K_{D}=2.20 \mu \mathrm{M}\right)$ (Table 1). This data suggests that while the loss of that $\mathrm{V}_{\mathrm{H}}-\mathrm{V}_{\mathrm{L}}$ interface hydrogen bond is important even for the UCA in gaining breadth against different viruses, the contribution from CDRs is still important.

\section{$\mathbf{V}_{\mathbf{H}}-\mathbf{V}_{\mathbf{L}}$ Interface Mutations Alter Relative Orientations of the Antibody Variable Domains}

Because $\mathrm{Q} 38_{\mathrm{L}}$ and $\mathrm{L} 46_{\mathrm{L}}$ of the light chain are involved in significant interactions at the $\mathrm{V}_{\mathrm{H}}-\mathrm{V}_{\mathrm{L}}$ interface and mutating these residues improves binding to Envs, we wanted to determine what structural changes these mutations cause. To aid in crystallization, we added an additional mutation, $\mathrm{Y} 32_{\mathrm{L}} \mathrm{N}$, in the light chain of I32M to create "I33M." Y32 $\mathrm{L}$ N occurs during 
A

13.2 Binding to $\mathrm{CH} 505$ gp120 V5 ETF Mutant

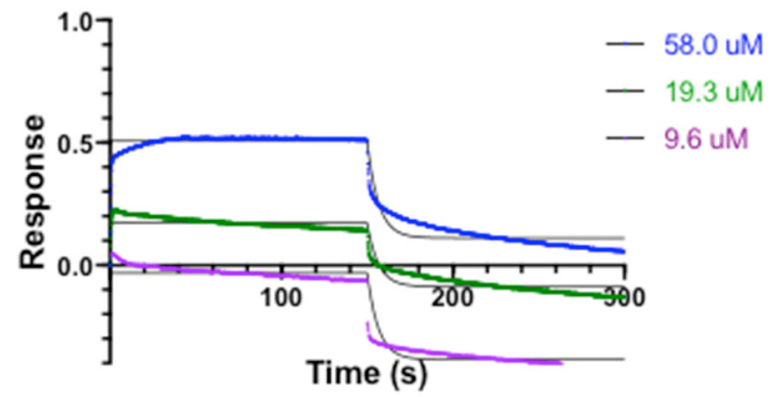

B

I32M Binding to $\mathrm{CH} 505$ gp120 V5 ETF Mutant

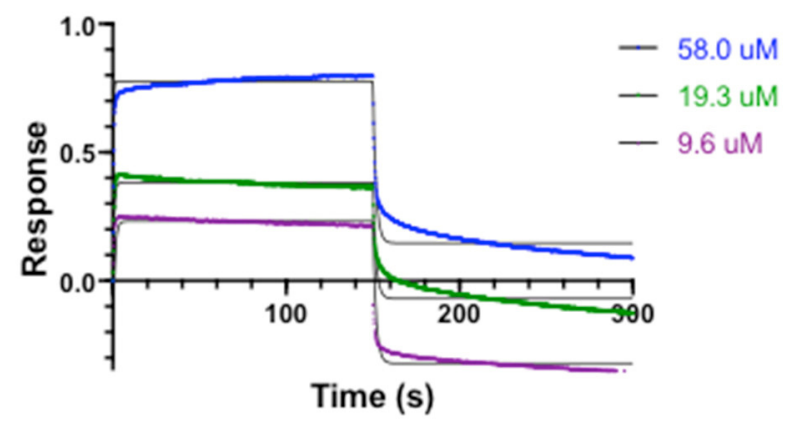

C

UCA Q39 $9_{\mathrm{H}} \mathrm{L}$ Binding to $92 \mathrm{UG} 037.8$ gp120 V5 mutant

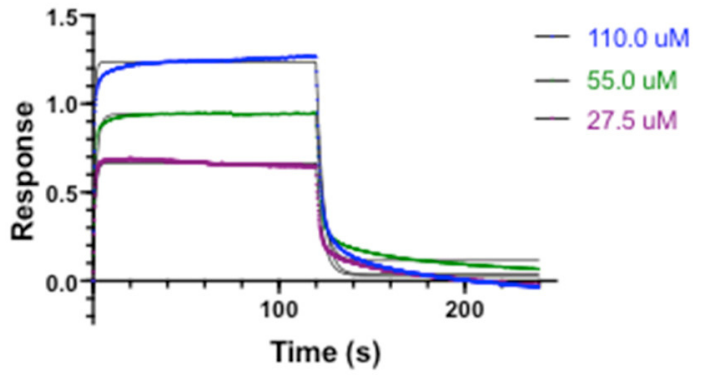

13.2 Binding to 92UG037.8 gp120 core

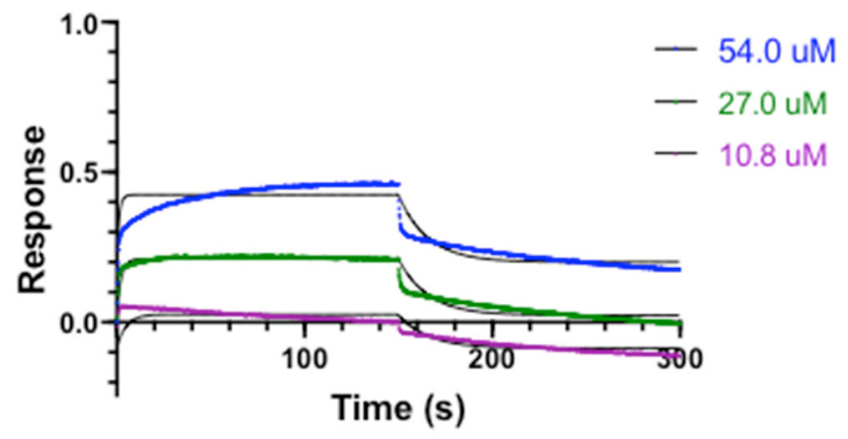

I32M Binding to 92UG037.8 gp120 core

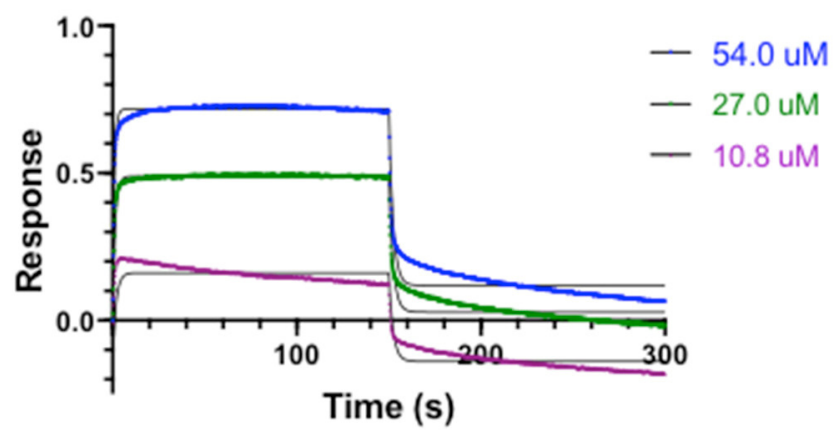

UCA WT Binding to 92UG037.8 gp120 V5 mutant

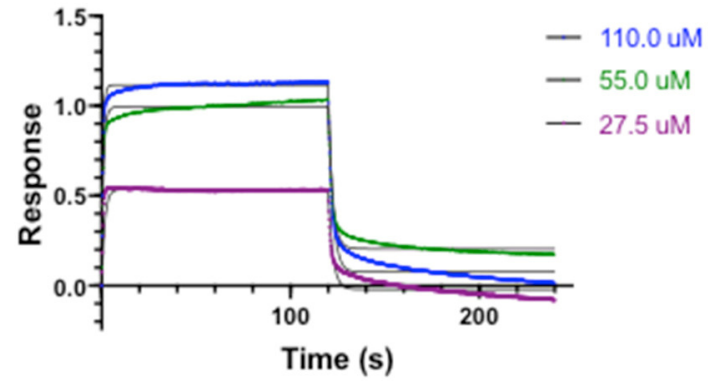

FIGURE 2 | Kinetic Studies by biolayer interferometry. Curves of I3.2 and I32M binding to (A) CH505 gp120 core with an "ETF" insertion in the V5 loop and (B) 92UG037.8 gp120 core. (C) Curves of the UCA and the UCA Q39HL mutant binding to 92UG037.8 gp120 core with a V5 mutation. The V5 loop of CH505 T/F Env was grafted in the place of the V5 loop of 92UG037.8 Env. In each case, the Fab was immobilized onto an anti-human Fab-CH1 biosensor, and gp120 constructs were introduced at three or more different concentrations, ranging from low micromolar to high micromolar, depending on the mutant tested.

affinity maturation of the $\mathrm{CH} 103$ lineage and N32 $\mathrm{L}$ first appears in the $\mathrm{V}_{\mathrm{L}} \mathrm{J}_{\mathrm{L}}$ of intermediate antibody $\mathrm{I} 2$ (Figures $\mathbf{1 B}, \mathrm{C}$ ). In later members of the lineage, $\mathrm{N} 32_{\mathrm{L}}$ of the light chain $\mathrm{CDR}$ loop 1 (LCDR1) was observed to hydrogen bond with the LCDR2 and HCDR3 loops, stabilizing the conformation of the latter and so we hypothesized the same would happen in I33M.
We determined the crystal structure of I33M to a resolution of $2.36 \AA$ (Table 2) and compared it to those of the wildtype intermediate $\mathrm{I} 3.2$, the $\mathrm{CH} 103$ bnAb, and chimeric I3.1 Fabs (Figure 3, Supplementary Figure 3). The $\mathrm{V}_{\mathrm{H}}$ of I33M was superposed on that of the wild-type Fabs, since the $\mathrm{V}_{\mathrm{H}}$ contacts the CD4bs on Env (Figure 1), which is invariant throughout the evolution of the virus and thus expected to bind antibodies 
TABLE 2 | Data collection and refinement statistics.

I33M

\begin{tabular}{|c|c|}
\hline \multicolumn{2}{|l|}{ Data collection } \\
\hline Space group & $P 6_{5}$ \\
\hline \multicolumn{2}{|l|}{ Cell dimensions } \\
\hline$a, b, c(\AA)$ & $131.6,131.6,104.9$ \\
\hline$\alpha, \beta, \gamma\left({ }^{\circ}\right)$ & $90,90,120$ \\
\hline Resolution ( $\AA$ ) & $50.0-2.36(2.40-2.36)$ \\
\hline Total reflections & 249,141 \\
\hline Unique reflections & 42,585 \\
\hline Completeness (\%) & $99.2(95.7)$ \\
\hline$R_{\text {merge }}$ & $12.6(97.4)$ \\
\hline $1 / \sigma(l)$ & $13.0(1.3)$ \\
\hline Multiplicity & $5.8(5.5)$ \\
\hline $\mathrm{CC}(1 / 2)$ & $0.993(0.504)$ \\
\hline \multicolumn{2}{|l|}{ Refinement } \\
\hline Resolution (Å) & $47.65-2.36$ \\
\hline$R_{\text {work }} / R_{\text {free }}(\%)$ & $18.5 / 22.3$ \\
\hline \multicolumn{2}{|l|}{ No. atoms } \\
\hline Protein & 6,346 \\
\hline Ligand/ion & 13 \\
\hline Water & 304 \\
\hline Ramachandran favored (\%) & 97 \\
\hline Ramachandran outliers (\%) & 1.3 \\
\hline \multicolumn{2}{|l|}{ R.M.S. deviations } \\
\hline Bond lengths ( $(\AA)$ & 0.008 \\
\hline Bond angles $\left({ }^{\circ}\right)$ & 1.15 \\
\hline \multicolumn{2}{|l|}{$B$-factors $\left(\AA^{2}\right)$} \\
\hline Protein & 52.70 \\
\hline Ligand/ion & 22.30 \\
\hline Water & 56.00 \\
\hline
\end{tabular}

*Values in parentheses are for highest-resolution shell.

in the same way throughout antibody affinity maturation. Superimposed $\mathrm{V}_{\mathrm{H}}$ domains of I3.2, I33M, and I3.1 demonstrate that $\mathrm{I} 33 \mathrm{M}$ has a $\mathrm{V}_{\mathrm{L}}$ orientation intermediate between that of I3.2 and I3.1 (Figures 3A-C), though it is much more similar to that of I3.1 and to mature members of the antibody lineage (Figure 3B, Supplementary Figure 3A). Additionally, the DE loop of the antibody, from which residues $65_{\mathrm{L}}-67_{\mathrm{L}}$ clash with the Env V5 loop in the case of I3.2 and the UCA of the lineage, is shifted for I33M in a way that would eliminate the clash, although it is not shifted to the same extent as I3.1 and the CH103 bnAb (Figure 3D, Supplementary Figure 3B).

As expected, the Q38 $\mathrm{L}$ V mutation in $\mathrm{I} 33 \mathrm{M}$ disrupted the reciprocal side-chain hydrogen bond interaction with $\mathrm{Q} 39_{\mathrm{H}}$, resulting in more compact hydrophobic packing seen in the mature CH103 lineage Fabs (Figure 4, Supplementary Figure 3). Moreover, in the case of the UCA and I3.2, L46 $\mathrm{L}$ is involved in hydrophobic packing by bearing on the $\mathrm{F} 100 \mathrm{D}_{\mathrm{H}}$ ring, which is a bridge between $\mathrm{P} 96_{\mathrm{H}}$ and $\mathrm{Y} 49_{\mathrm{L}}$ at the domain interface. Replacing leucine with the shorter valine at this position in $\mathrm{I} 33 \mathrm{M}$ causes $\mathrm{F}_{100 \mathrm{D}_{\mathrm{H}}}\left(\mathrm{Y}_{100 \mathrm{D}_{\mathrm{H}}}\right.$ in the case of the $\mathrm{CH} 103$ bnAb) to swing closer to $\mathrm{Y} 49_{\mathrm{L}}$ to maintain hydrophobic interactions, while
P96 $\mathrm{H}$ appears unaffected. This is similar to what was observed in I3.1 and the CH103 bnAb (Figure 4, Supplementary Figure 3C) (22). Thus, $\mathrm{Q} 38_{\mathrm{L}} \mathrm{V}$ and $\mathrm{L} 46_{\mathrm{L}} \mathrm{V}$ contribute to $\mathrm{V}_{\mathrm{H}}-\mathrm{V}_{\mathrm{L}}$ packing.

We further analyzed the structures to see if $\mathrm{N} 32_{\mathrm{L}}$ made similar contacts in the different antibodies despite the different extents of orientation shifts of their $\mathrm{V}_{\mathrm{L}}$ domains relative to $\mathrm{V}_{\mathrm{H}}$. As with the chimeric I3.1 and mature $\mathrm{CH} 103$ bnAb, N32 $\mathrm{L}$ in I33M could form the same hydrogen bond with the HCDR3 loop, specifically

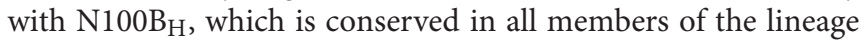
(Figure 4, Supplementary Figures 1, 3C). Of note, the distances between the backbones of light chain residue 32 (Y32 $\mathrm{L}$ in the case of the early CH103-lineage members UCA and I3.2 and N32L for later members and $\mathrm{I} 33 \mathrm{M}$ ) and heavy chain residue $100 \mathrm{~B}$ are very similar for the three Fabs: $6.4 \AA$ for I33M, 6.2 $\AA$ for I3.1, and 6.4 $\AA$ for I3.2, suggesting that the $\mathrm{Y} 32_{\mathrm{L}} \mathrm{N}$ mutation does not alter the $\mathrm{V}_{\mathrm{H}}-\mathrm{V}_{\mathrm{L}}$ domain interface spacing and that the $\mathrm{Q} 38_{\mathrm{L}} \mathrm{V}$ and $\mathrm{L} 46_{\mathrm{L}} \mathrm{V}$ mutations are the sole determinants of the observed shift.

To determine what effect $\mathrm{Y} 32_{\mathrm{L}} \mathrm{N}$ has on binding Envs, we performed BLI with $\mathrm{I} 33 \mathrm{M}$ and found that it bound with a stronger affinity to the CH505 gp120 T/F core ETF mutant and 92UG037.8 wild-type gp120 core than I32M but with the same affinity to CH505 gp120 T/F wild-type gp120 core (Table 1). This suggests that the hydrogen bond that N32 L makes with the HCDR3 loop, which binds to the CD4bs, contributes to Env binding.

\section{$\mathbf{V}_{\mathbf{H}}-\mathbf{V}_{\mathrm{L}}$ Interface Mutations Do Not Alter Antibody Stability}

To determine what, if any, effects the $\mathrm{V}_{\mathrm{H}}-\mathrm{V}_{\mathrm{L}}$ interface mutations and structural changes have on thermostability, we measured the melting temperature $\left(T_{m}\right)$ of the mutants we generated using circular dichroism thermal melts (Figure 5). $T_{m}$ values for our mutants are similar and fall within the wide reported range of Fab melting temperatures $\left(65-90^{\circ} \mathrm{C}\right)(26,27)$. Comparison to the melting temperature of I3.2 shows that the introduction of mutations in the light chain at the $\mathrm{V}_{\mathrm{H}}-\mathrm{V}_{\mathrm{L}}$ interface, or $\mathrm{Y} 32_{\mathrm{L}} \mathrm{N}$ in the CDR, has no significant effect on thermostability. We also determined the $T_{m}$ for the $\mathrm{CH} 103$ bnAb. $\mathrm{CH} 103$ had a similar melting temperature as I3.2, consistent with the reported value of $65.5^{\circ} \mathrm{C}$ and $T_{m}$ trend determined by differential scanning calorimetry by others (18).

\section{DISCUSSION}

HIV-1 bnAbs that evolve naturally in infected individuals have high levels of mutations in the CDRs and FWR. While many mutations in the CDR can impact binding to antigen, the roles of the FWR mutations are usually less clear. It has been shown that FWR mutations can be important for the development of breadth $(18,28)$. In this study, we identified two FWR residues at the $\mathrm{V}_{\mathrm{H}}-\mathrm{V}_{\mathrm{L}}$ interface, $\mathrm{Q} 38_{\mathrm{L}} \mathrm{V}$ and $\mathrm{L} 46_{\mathrm{L}} \mathrm{V}$, that were important for improving binding to HIV-1 Envs with longer V5 loops. These two mutations alone were sufficient for the significant shift of the orientation of the $\mathrm{V}_{\mathrm{L}}$ domain with respect to $\mathrm{V}_{\mathrm{H}}$ over the course of affinity maturation of the $\mathrm{CH} 103$ bnAb lineage. Moreover, 
A

$$
\text { Antigen Binding Site }
$$

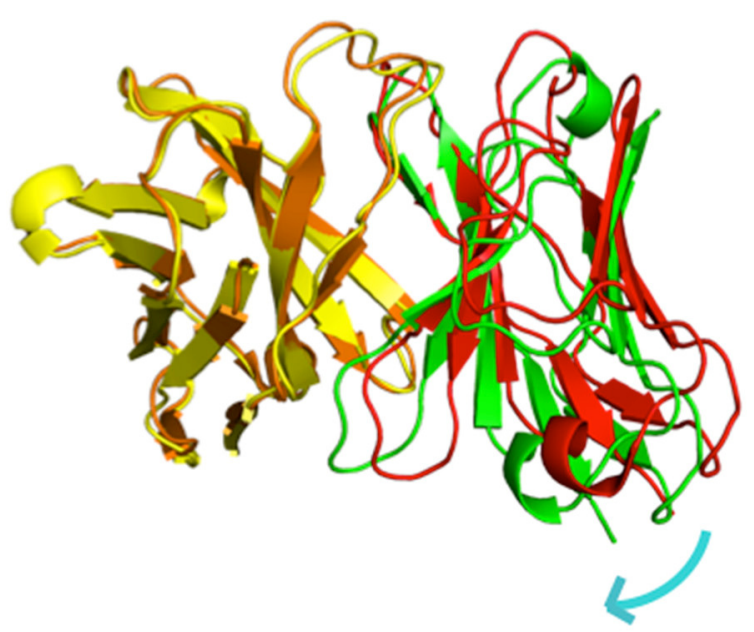

C

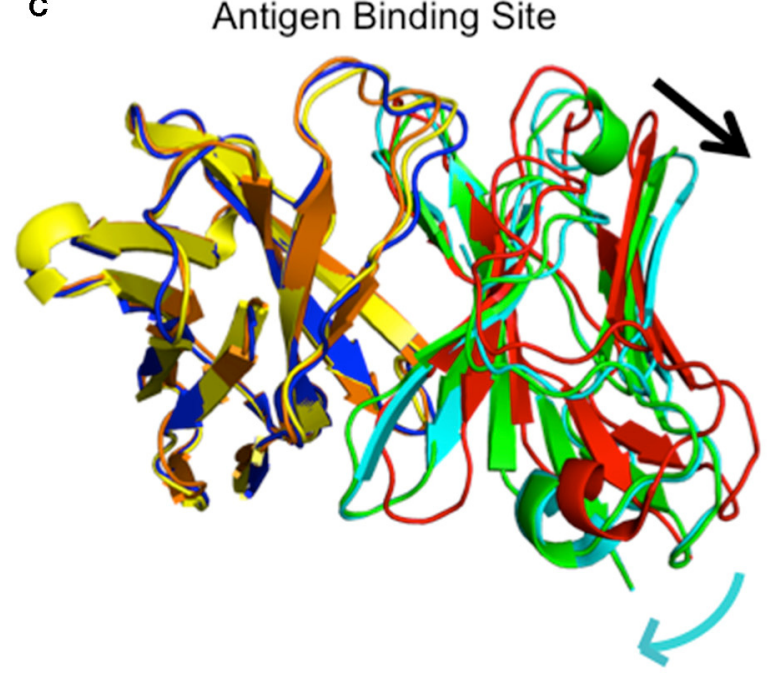

B Antigen Binding Site

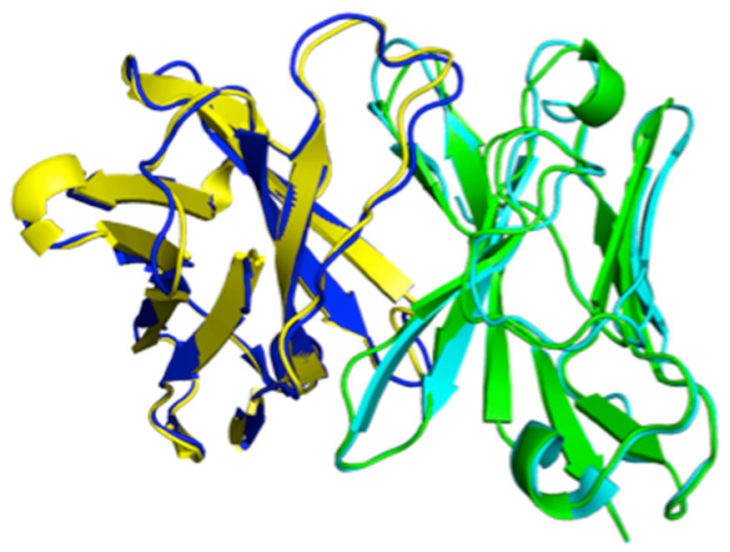

D

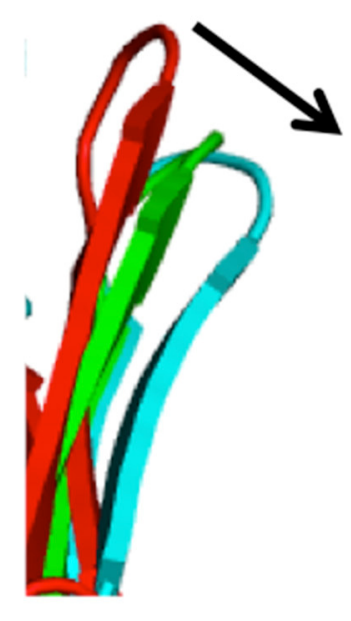

FIGURE 3 | Superpositions of I3.2, I33M, and chimeric 13.1. (A) Overall superposition of mutant Fab I33M onto I3.2. The domains are colored: I33M $V_{H}$ (yellow) and $V_{\mathrm{L}}$ (green), and I3.2 $\mathrm{V}_{\mathrm{H}}$ (orange) and $\mathrm{V}_{\mathrm{L}}$ (red). (B) Overall superposition of mutant Fab I33M onto I3.1. The domains are colored: I33M $\mathrm{V}_{\mathrm{H}}$ (yellow) and $\mathrm{V}_{\mathrm{L}}$ (green), and I3.1 $\mathrm{V}_{\mathrm{H}}$ (blue) and $\mathrm{V}_{\mathrm{L}}$ (cyan). (C) Overall superposition of mutant Fab I33M onto I3.2 and I3.1. Domains are colored as in panels (A,B). The constant domains are not shown for clarity. Relative shifts are indicated with a cyan arrow. The black arrow shows the shift in the antibody DE loop. (D) Zoomed in view of the DE loops of I3.2 (red), I33M (green), and I3.1 (cyan).

these mutations did not alter the stability of the antibodies consistent with reports elsewhere (18).

Q38 $\mathrm{L}$, distal to the Env binding site, eliminates hydrogen bonding with $\mathrm{Q} 39_{\mathrm{H}}$. Moreover, the $\mathrm{Q} 39_{\mathrm{H}} \mathrm{L}$ heavy chain mutation first appeared in I2 of the lineage and so I3.2 has a Q39 $\mathrm{H}$ (Supplementary Figure 1). Our structure of I33M shows that a relative shift in orientation of the domains can occur in the absence of the $\mathrm{Q} 39_{\mathrm{H}} \mathrm{L}$ mutation, since this mutant only consisted of light chain mutations in I3.2 (Figure 4). Moreover, mutation of just one of these residues alone, which disrupts the variable interface hydrogen bond, is sufficient for improving binding to a heterologous HIV-1 Env (Figure 2C, Table 1), an important feature in the development of breadth. $\mathrm{L} 46_{\mathrm{L}} \mathrm{V}$, also within the $\mathrm{V}_{\mathrm{H}}-\mathrm{V}_{\mathrm{L}}$ interface, alters hydrophobic packing. Thus, the mutations $\mathrm{Q} 38_{\mathrm{L}} \mathrm{V}$ and $\mathrm{L} 46_{\mathrm{L}} \mathrm{V}$ in the antibody light chain brought the relative orientation of the $\mathrm{V}_{\mathrm{H}}$ and $\mathrm{V}_{\mathrm{L}}$ domains of the antibodies closer to that of the mature members of the $\mathrm{CH} 103$ lineage to accommodate Envs with larger V5 loops. Of note, the I32M double mutant bound to the CH505 gp120 core "ETF" V5 loop mutant with a similar dissociation constant (Table 1) as a later intermediate, I2 (22) and the chimeric I3.1. This suggests that all the mutations accrued in late $\mathrm{CH} 103$ lineage members may not be necessary for antibody affinity maturation, at least against autologous Envs. These results further demonstrate that it is important to consider more than just the CDRs for vaccine design. 


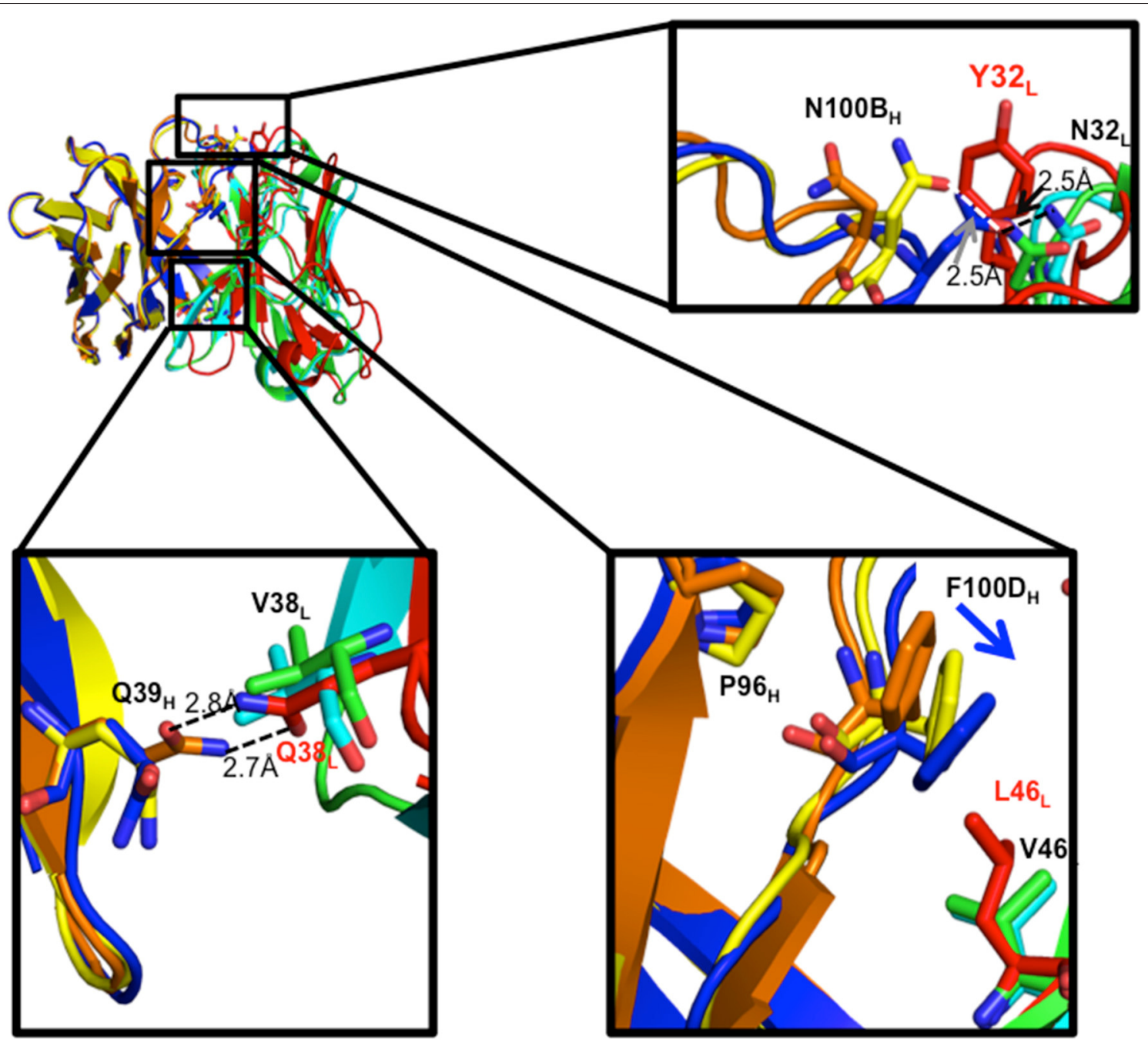

FIGURE $4 \mid V_{H}-V_{L}$ interface residues and their effects. Zoomed in views of residues that were mutated are shown as sticks for the three Fabs. Domains for the different Fabs are colored as follows: I33M $V_{H}$ (yellow) and $V_{L}$ (green), I3.2 $V_{H}$ (orange) and $V_{L}$ (red), and $I 3.1 V_{H}$ (blue) and $V_{L}$ (cyan). Hydrogen bond distances are given.

Our work did also identify a few critical CDR mutations that occurred throughout affinity maturation that greatly improved antibody binding to Env. A significant improvement in $K_{D}$ was observed when we introduced the $\mathrm{S} 56_{\mathrm{H}} \mathrm{E}$ mutation into the wild-type UCA ( $\sim 7$-fold) or the UCA Q39 ${ }_{\mathrm{H}} \mathrm{L}$ mutant $(\sim 3$-fold; Table 1). Another example of a single CDR mutation enhancing affinity was demonstrated by our I33M mutant, which had the Y $32_{\mathrm{L}} \mathrm{N}$ mutation in the LCDR1. The I33M mutant bound to the 92UG037.8 gp120 core with a $\sim 3$-fold lower $K_{D}$ than that of I32M (Table 1). A likely consequence of the Y $32_{\mathrm{L}} \mathrm{N}$ mutation in the LCDR1 of I33M and other mature CH103-lineage members is the stabilization of the configuration of the HCDR3 loop, important for contacting the conserved CD4bs of the CH505 HIV-1 Env. The HCDR3 loop was disordered in the UCA, but not in later members of the $\mathrm{CH} 103$ lineage (22). The hydrogen bonding we observed in $\mathrm{I} 33 \mathrm{M}$ between $\mathrm{N} 32_{\mathrm{L}}$ and $\mathrm{N} 100 \mathrm{~B}_{\mathrm{H}}$ (Figure 4) is reminiscent of what is observed in I2 and the bnAbs from this lineage. This illustrates that very few mutations were necessary for the correct configuration of the HCDR3 loop. While FWR mutations have been shown to increase antibody flexibility in some cases $(17,28)$, results from our crystal structure are consistent with more standard trends observed, i.e., that affinity maturation results in a more rigid antibody structure, specifically in the HCDR3 loop (8, 29-33).

In the $\mathrm{CH} 103$ lineage, a significant increase in both heterologous binding and heterologous neutralization was observed during the transition from I3.2 to I2, without much change thereafter (21). Additionally, a shift in the orientation of the $\mathrm{V}_{\mathrm{L}}$ domain relative to that of the $\mathrm{V}_{\mathrm{H}}$ was also observed between these two antibodies, and the $\mathrm{CH} 103$ bnAb had a similar $\mathrm{V}_{\mathrm{L}}$ orientation as $\mathrm{I} 2$ and the chimeric $\mathrm{I} 3.1$ with respect to $\mathrm{V}_{\mathrm{H}}$ (22). Furthermore, it was shown through structures and binding data, that $\mathrm{CH} 103$-lineage Fabs whose $\mathrm{V}_{\mathrm{L}}$ domain had shifted relative to the $\mathrm{V}_{\mathrm{H}}$ domain had higher binding affinity toward HIV Envs with longer V5 loops than antibodies from the lineage in which this shift had not yet occurred. Because many other 
74

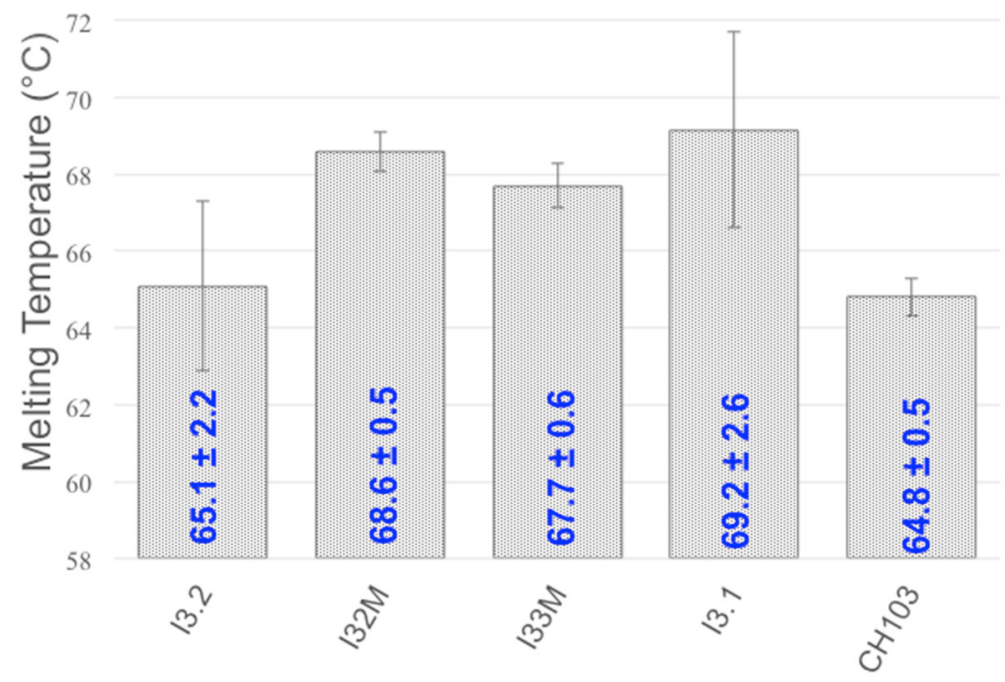

FIGURE 5 | Melting temperatures of Fabs. $T_{m}$ 's of various Fabs and mutants are shown. Standard deviations are from three or more replicates.

HIV Envs tend to have V5 loops that are longer than that of the CH505 T/F, this suggests there may be a correlation between a shift in $\mathrm{V}_{\mathrm{L}}$ and heterologous neutralization breadth in the case of the CH103 lineage. Since our I3.2 mutant also had a shift in $\mathrm{V}_{\mathrm{L}}$, we expect that I32M and I33M have broader neutralization capabilities than the wild-type I3.2, though this has yet to be confirmed experimentally.

A challenge in eliciting antibodies with great breadth via HIV-1 vaccination is the fact that bnAbs take years to develop, requiring high mutation frequencies. Thus, a deep understanding of antibody affinity maturation, paired with virus evolution, is needed to determine key steps in these processes and to identify which stages to target by vaccination. The relative shift in the $V_{H}$ and $\mathrm{V}_{\mathrm{L}}$ domains was demonstrated to be important and here we show that very few mutations in the antibody variable domain interface are necessary for this structural change. Thus, exposure to an antigen that would lead to such a shift may lead to a more rapid antibody response than one in which many mutations in the antigen combining site are necessary. Our results also have implications for the design of therapeutic antibodies. Alterations at the $\mathrm{V}_{\mathrm{H}}-\mathrm{V}_{\mathrm{L}}$ interface can alter the antigen-combining site in a favorable way to lead to more potent and broad antibodies.

Complex evolutionary trajectories are followed in different HIV-1 bnAb lineages, therefore, structural analyses at multiple time points during the maturation pathway could reveal new phenomena. Since the shift in orientation of $V_{L}$ relative to $V_{H}$ that we observed in our mutant is similar to but not exactly the same as that observed in the mature $\mathrm{CH} 103$ lineage members, there may be additional mutations that would result in a greater shift and should be explored. By comparing available structures of the wild-type, chimeric, and mutant antibodies (22), we find that there are no other mutations in the antibody light chain at the $\mathrm{V}_{\mathrm{H}}-\mathrm{V}_{\mathrm{L}}$ interface that change identity, rotamers, or amino acid binding partners throughout affinity maturation of this particular lineage. Thus, it is possible that there are mutations in other regions of the FWR or in the CDRs that affect the shift in $\mathrm{V}_{\mathrm{L}}$ relative to $\mathrm{V}_{\mathrm{H}}$. Additionally, a high-resolution structure of the CH103 UCA in complex with an Env has not yet been determined. Such a structure is necessary to better understand antibody-virus co-evolution in this lineage as well as properties of HIV-1 Env that triggered the lineage. While the CH103 UCA can only bind the CH505 T/F Env, it binds poorly to an Env stabilized in a closed conformation (data not shown). Our work shows that introducing the $\mathrm{S} 56_{\mathrm{H}} \mathrm{E}$ mutation in the UCA HCDR2 loop alone significantly improves its binding to a heterologous Env (Table 1). This provides a means for determining an atomic resolution structure of the UCA with Env to provide greater insights for HIV-1 vaccine development.

\section{MATERIALS AND METHODS \\ Expression and Purification of Fabs}

Site-directed mutagenesis was performed using manufacturer's protocols (Stratagene) to introduce mutations into the UCA heavy and light chains. Fabs were expressed in HEK293F suspension adapted cells via transient transfection using linear polyethylenimine (PEI) following the manufacturer's suggested protocol. For $200 \mathrm{~mL}$ of suspension cell culture, $100 \mu \mathrm{g}$ of total plasmid was used. After 5 days of expression, the supernatant was clarified by centrifugation. Each His-tagged CH103-lineage Fab was loaded onto Ni-NTA superflow resin (Qiagen) preequilibrated with Buffer A (10 mM Tris, pH 7.5, $200 \mathrm{mM} \mathrm{NaCl}$ ), washed with Buffer A then Buffer $\mathrm{A}+10 \mathrm{mM}$ imidazole, and finally, eluted with Buffer A + 350mM imidazole. Each Fab was then purified by gel filtration chromatography using a Superdex ${ }^{\mathrm{TM}} 200$ Increase 10/300 GL column (GE Healthcare) in 
buffer of $10 \mathrm{mM}$ Tris, $\mathrm{pH} 7.5,100 \mathrm{mM} \mathrm{NaCl}$. Fractions of interest were further concentrated.

Plasmids containing the CMVR VRC01 Ig heavy and light chains were obtained from the NIH Aids Reagents Program. The protein was expressed in $293 \mathrm{~F}$ cells as described for Fabs above. The clarified supernatant was diluted twofold using $1 \times$ PBS buffer and purified using protein A agarose resin (Pierce), according to manufacturer's protocols. The eluent was concentrated and further purified by gel filtration chromatography in buffer of $10 \mathrm{mM}$ Tris, $\mathrm{pH} 7.5,100 \mathrm{mM}$ $\mathrm{NaCl}$ using a Superdex ${ }^{\mathrm{TM}} 200$ Increase $10 / 300$ GL column (GE Healthcare). The VRC01 Fab was obtained by digesting the Ig using papain (Pierce) and by running the digested products through protein A resin (Pierce) according to manufacturer's protocol. The Fab was then purified by gel filtration chromatography again as described above.

\section{Expression and Purification of HIV-1 Env gp120s}

The codon-optimized synthetic constructs of the CH505 T/F HIV-1 subtype C gp120 containing amino acid (a.a.) 41-492 (HXB2 numbering) $\triangle \mathrm{V} 123$ (core) was described previously (22). Briefly, the same pVRC-8400 expression vector as described for Fabs was used and the construct contained an N-terminal $6 \times$-histidine tag. The expression construct also contained a leader sequence encoding the tissue plasminogen activator signal sequence. The IRES-puro vector containing 92UG037.8 HIV-1 subtype A gp120 a.a. residues 1-492 (HXB2 numbering) $\Delta$ V123 (core) with a $6 \times$-histidine tag inserted between residues 40 and 41 and V5 mutations was also described previously (22). Recombinant Env glycoproteins were expressed in HEK 293T cells and purified as described for His-tagged Fabs.

\section{Bio-layer Interferometry}

Kinetic measurements of Fab binding to HIV-1 Env gp120 cores were carried out using the fortéBIO BLItz instrument using the same buffer all of our proteins were purified in $(10 \mathrm{mM}$ Tris, pH 7.5, 100mM NaCl) to minimize bulk shift effects. Fabs at concentration $0.2 \mathrm{mg} / \mathrm{mL}$ were immobilized on Dip and Read $^{\mathrm{TM}}$ Anti-Human Fab-CH1 second Generation Biosensors until saturation was reached (3 min). Env constructs were tested at concentrations ranging from 9.6 to $110 \mu \mathrm{M}$, depending on the particular construct used and its solubility, as well as based on its binding affinity to each Fab tested. The CH505 T/F gp120 wild-type core was tested at concentrations of 6.8, 22.0, and $66.0 \mu \mathrm{M}$, the CH505 T/F gp120 core ETF mutant was tested at concentrations 9.6, 19.3, and $58.0 \mu \mathrm{M}$, the 92UG037.8 gp120 core was tested at concentrations $10.8,27$, and $54.0 \mu \mathrm{M}$, and the 92UG037.8 gp120 core V5 mutant was tested at concentrations 27.5, 55.0, and $110.0 \mu \mathrm{M}$. Association and dissociation were each monitored for $2-2.5 \mathrm{~min}$. Step corrections, at the start of association and at the start of dissociation, were performed manually using Microsoft Excel. Analyses were then performed using a global fit of at least three measurements using nonlinear regression curve fitting using the Graphpad Prism software, version 8.

\section{Circular Dichroism}

CD spectra were recorded over the range of $260-210 \mathrm{~nm}$ to confirm the presence of a $\beta$-sheet signal at $216 \mathrm{~nm}$ (34) using an AVIV Model 435 Circular Dichroism Spectrometer. Fabs were loaded into $700 \mu \mathrm{L}$ quartz crystal cuvettes at a concentration of $0.02 \mathrm{mg} / \mathrm{mL}$ in PBS buffer. Thermal melts were done from 20 to $90^{\circ} \mathrm{C}$. CD signals at $216 \mathrm{~nm}$ were monitored every $0.5^{\circ} \mathrm{C}$ as the temperature of the samples increased at a ramp rate of $0.5^{\circ} \mathrm{C} / \mathrm{min}$. The samples were allowed to equilibrate for $5 \mathrm{~s}$ at each temperature before $\mathrm{CD}$ signals were recorded at an averaging time of $15 \mathrm{~s}$. Data was analyzed with OriginLab using signal smoothing and assuming a two-state model with constant $\Delta H$ (35). In order to obtain the best fits, the approximated starting and ending baselines (which were assumed to be linear), melting temperature, and enthalpy of unfolding were adjusted. Curves were fit to a chi-squared tolerance value of $1 \times 10^{-9}$.

\section{Crystallization of $\mathrm{CH} 103$ Lineage Fab Mutants}

I33M was concentrated to $11.24 \mathrm{mg} / \mathrm{mL}$ prior to crystallization. I33M crystallized in 96-well-tray format, set up with a TTPLabTech Mosquito ${ }^{\circledR} \mathrm{HTS}$ in $1.5 \mathrm{M} \mathrm{AmSO}_{4}, 100 \mathrm{mM}$ Hepes, pH 7.0, 5\% PEG 400 and was optimized in 24-well-format in which the $\mathrm{pH}$ of the solution was adjusted to 7.23.

\section{Data Collection, Structure Determination, and Refinement}

$\mathrm{X}$-ray diffraction data was collected using beamlines at the Advanced Photon Source at Argonne National Laboratory. I33M data were collected at beam line 24-ID-E using a $0.98 \AA$ wavelength. Data set from an individual crystal was processed with HKL2000 (36). Molecular replacement calculations for the Fab were carried out with PHASER (37) using published I3.2 [Protein Data Bank (PDB) ID 4QHL] as the search model. The Fab was broken up into the variable and constant domains in order to perform two separate searches. I33M contained two molecules in the asymmetric subunit.

Refinement was carried out with PHENIX (38), and all model modifications were carried out with Coot (39) and PyMOL. During refinement, maps were generated from combinations of positional, group B-factor, and TLS (translation/libration/screw) refinement algorithms. All refinements used secondary structure and NCS restraints. Structure validations were performed periodically during refinement using the MolProbity server (40). The final refinement statistics are summarized in Table 2.

\section{Protein Structure Analysis and Graphical Representations}

The structures analyzed in this study were superposed by least squares fitting in Coot.

\section{DATA AVAILABILITY STATEMENT}

The datasets generated for this study can be found in the Protein Data Bank accession code 6VPY. 


\section{AUTHOR CONTRIBUTIONS}

JZ and DF coordinated and designed the study, analyzed and evaluated the data, and wrote and edited the manuscript and figures. JZ, HZ, TT, and DF performed experiments: TT, JZ, and DF expressed and purified proteins. TT and HZ performed BLI experiments. JZ collected CD spectra and performed thermal melts. JZ and DF crystallized proteins and determined structures. All authors contributed to the article and approved the submitted version.

\section{FUNDING}

JZ was supported by the Meyer Davidson '57 Summer Research Fellowship Grant. DF acknowledges support from the Mathilde Krim Fellowship in Basic Biomedical Research (109502-61RKVA) from amfAR and Swarthmore College Startup and Faculty Research Funds. This work is based upon research conducted at NE-CAT beamlines, which are funded by the NIGMS from the NIH (P30 GM124165). The Eiger 16M detector

\section{REFERENCES}

1. Mishra AK, Mariuzza RA. Insights into the structural basis of antibody affinity maturation from next-generation sequencing. Front Immunol. (2018) 9:117. doi: 10.3389/fimmu.2018.00117

2. Binley JM, Lybarger EA, Crooks ET, Seaman MS, Gray E, Davis KL, et al. Profiling the specificity of neutralizing antibodies in a large panel of plasmas from patients chronically infected with human immunodeficiency virus type 1 subtypes B and C. J Virol. (2008) 82:11651-68. doi: 10.1128/JVI.01762-08

3. Wu NC, Wilson IA. Structural insights into the design of novel anti-influenza therapies. Nat Struct Mol Biol. (2018) 25:115-21. doi: 10.1038/s41594-018-0025-9

4. Richman DD, Wrin T, Little SJ, Petropoulos CJ. Rapid evolution of the neutralizing antibody response to HIV type 1 infection. Proc Natl Acad Sci US A. (2003) 100:4144-9. doi: 10.1073/pnas.0630530100

5. Wei X, Decker JM, Wang S, Hui H, Kappes JC, Wu X, et al. Antibody neutralization and escape by HIV-1. Nature. (2003) 422:307-12. doi: $10.1038 /$ nature 01470

6. Doria-Rose NA, Schramm CA, Gorman J, Moore PL, Bhiman JN, DeKosky BJ, et al. Developmental pathway for potent V1V2-directed HIV-neutralizing antibodies. Nature. (2014) 509:55-62. doi: 10.1038/nature13036

7. Davenport TM, Gorman J, Joyce MG, Zhou T, Soto C, Guttman M, et al. Somatic hypermutation-induced changes in the structure and dynamics of HIV-1 broadly neutralizing antibodies. Structure. (2016) 24:1346-57. doi: 10.1016/j.str.2016.06.012

8. Schmidt AG, Xu H, Khan AR, O’Donnell T, Khurana S, King LR, et al. Preconfiguration of the antigen-binding site during affinity maturation of a broadly neutralizing influenza virus antibody. Proc Natl Acad Sci U S A. (2013) 110:264-9. doi: 10.1073/pnas.1218256109

9. Bonsignori M, Kreider EF, Fera D, Meyerhoff RR, Bradley T, Wiehe K, et al. Staged induction of HIV-1 glycan-dependent broadly neutralizing antibodies. Sci Transl Med. (2017) 9:eaai7514. doi: 10.1126/scitranslmed.aai7514

10. Fera D, Lee MS, Wiehe K, Meyerhoff RR, Piai A, Bonsignori M, et al. HIV envelope V3 region mimic embodies key features of a broadly neutralizing antibody lineage epitope. Nat Commun. (2018) 9:1111. doi: 10.1038/s41467-018-03565-6

11. Saunders KO, Wiehe K, Tian M, Acharya P, Bradley T, Alam SM, et al. Targeted selection of HIV-specific antibody mutations by engineering B cell maturation. Science. (2019) 366:eaay7199. doi: 10.1126/science.aay7199

12. Wiehe K, Bradley T, Meyerhoff RR, Hart C, Williams WB, Easterhoff D, et al. Functional relevance of improbable antibody mutations for HIV broadly on the 24-ID-E beam line is funded by a NIH-ORIP HEI grant (S10OD021527). This research used resources of the Advanced Photon Source, a U.S. Department of Energy (DOE) Office of Science User Facility operated for the DOE Office of Science by Argonne National Laboratory under Contract No. DE-AC02$06 \mathrm{CH} 11357$.

\section{ACKNOWLEDGMENTS}

We thank staff at Northeastern Collaborative Access Team (NE-CAT) X-Ray beam lines $24 \mathrm{ID}-\mathrm{C} / \mathrm{E}$ (Advanced Photon Source). We thank Liliya Yatsunyk and Deondre Jordan for advice on the $\mathrm{CD}$ experiments. We thank the laboratory of Stephen Harrison for providing plasmids.

\section{SUPPLEMENTARY MATERIAL}

The Supplementary Material for this article can be found online at: https://www.frontiersin.org/articles/10.3389/fimmu. 2020.01529/full\#supplementary-material neutralizing antibody development. Cell Host Microbe. (2018) 23:759-65.e6. doi: 10.1016/j.chom.2018.04.018

13. Sok D, Laserson U, Laserson J, Liu Y, Vigneault F, Julien JP, et al. The effects of somatic hypermutation on neutralization and binding in the PGT121 family of broadly neutralizing HIV antibodies. PLoS Pathog. (2013) 9:e1003754. doi: 10.1371/journal.ppat.1003754

14. Jardine JG, Sok D, Julien JP, Briney B, Sarkar A, Liang CH, et al. Minimally mutated HIV-1 broadly neutralizing antibodies to guide reductionist vaccine design. PLoS Pathog. (2016) 12:e1005815. doi: 10.1371/journal.ppat.1005815

15. Garces F, Lee JH, de Val N, de la Pena AT, Kong L, Puchades C, et al. Affinity maturation of a potent family of HIV antibodies is primarily focused on accommodating or avoiding glycans. Immunity. (2015) 43:105363. doi: $10.1016 /$ j.immuni.2015.11.007

16. Garces F, Sok D, Kong L, McBride R, Kim HJ, Saye-Francisco KF, et al. Structural evolution of glycan recognition by a family of potent HIV antibodies. Cell. (2014) 159:69-79. doi: 10.1016/j.cell.2014.09.009

17. Klein F, Diskin R, Scheid JF, Gaebler C, Mouquet H, Georgiev IS et al. Somatic mutations of the immunoglobulin framework are generally required for broad and potent HIV-1 neutralization. Cell. (2013) 153:126-38 doi: 10.1016/j.cell.2013.03.018

18. Henderson R, Watts BE, Ergin HN, Anasti K, Parks R, Xia S-M, et al. Selection of immunoglobulin elbow region mutations impacts interdomain conformational flexibility in HIV-1 broadly neutralizing antibodies. Nat Commun. (2019) 10:654. doi: 10.1038/s41467-019-08415-7

19. Koenig P, Lee CV, Walters BT, Janakiraman V, Stinson J, Patapoff TW, et al. Mutational landscape of antibody variable domains reveals a switch modulating the interdomain conformational dynamics and antigen binding. Proc Natl Acad Sci U S A. (2017) 114:E486-E95. doi: 10.1073/pnas.161 3231114

20. Ovchinnikov V, Louveau JE, Barton JP, Karplus M, Chakraborty AK. Role of framework mutations and antibody flexibility in the evolution of broadly neutralizing antibodies. Elife. (2018) 7:e33038. doi: 10.7554/eLife.33038

21. Liao HX, Lynch R, Zhou T, Gao F, Alam SM, Boyd SD, et al. Co-evolution of a broadly neutralizing HIV-1 antibody and founder virus. Nature. (2013) 496:469-76. doi: 10.1038/nature12053

22. Fera D, Schmidt AG, Haynes BF, Gao F, Liao HX, Kepler TB, et al. Affinity maturation in an HIV broadly neutralizing B-cell lineage through reorientation of variable domains. Proc Natl Acad Sci U S A. (2014) 111:1027580. doi: 10.1073/pnas.1409954111

23. Pietzsch J, Scheid JF, Mouquet H, Klein F, Seaman MS, Jankovic M et al. Human anti-HIV-neutralizing antibodies frequently target a conserved 
epitope essential for viral fitness. J Exp Med. (2010) 207:1995-2002. doi: $10.1084 / \mathrm{jem} .20101176$

24. Li Y, O’Dell S, Walker LM, Wu X, Guenaga J, Feng Y, et al. Mechanism of neutralization by the broadly neutralizing HIV-1 monoclonal antibody VRC01. J Virol. (2011) 85:8954-67. doi: 10.1128/JVI.00754-11

25. Zhou T, Georgiev I, Wu X, Yang ZY, Dai K, Finzi A, et al. Structural basis for broad and potent neutralization of HIV-1 by antibody VRC01. Science. (2010) 329:811-7. doi: 10.1126/science.1192819

26. McConnell AD, Zhang X, Macomber JL, Chau B, Sheffer JC, Rahmanian S, et al. A general approach to antibody thermostabilization. MAbs. (2014) 6:1274-82. doi: 10.4161/mabs.29680

27. Wozniak-Knopp G, Stadlmayr G, Perthold JW, Stadlbauer K, Gotsmy M, Becker S, et al. An antibody with Fab-constant domains exchanged for a pair of CH3 domains. PLoS One. (2018) 13:e0195442. doi: 10.1371/journal.pone. 0195442

28. Scheid JF, Mouquet H, Ueberheide B, Diskin R, Klein F, Oliveira TY, et al. Sequence and structural convergence of broad and potent HIV antibodies that mimic CD4 binding. Science. (2011) 333:1633-7. doi: 10.1126/science.1207227

29. Eisen HN, Chakraborty AK. Evolving concepts of specificity in immune reactions. Proc Natl Acad Sci U S A. (2010) 107:22373-80. doi: $10.1073 /$ pnas. 1012051108

30. Foote J, Milstein C. Conformational isomerism and the diversity of antibodies. Proc Natl Acad Sci U S A. (1994) 91:10370-4. doi: 10.1073/pnas.91.22.10370

31. Worn A, Pluckthun A. Mutual stabilization of VL and VH in singlechain antibody fragments, investigated with mutants engineered for stability. Biochemistry. (1998) 37:13120-7. doi: 10.1021/bi980712q

32. Julian MC, Li L, Garde S, Wilen R, Tessier PM. Efficient affinity maturation of antibody variable domains requires co-selection of compensatory mutations to maintain thermodynamic stability. Sci Rep. (2017) 7:45259. doi: $10.1038 /$ srep 45259

33. Wedemayer GJ, Patten PA, Wang LH, Schultz PG, Stevens RC. Structural insights into the evolution of an antibody combining site. Science. (1997) 276:1665-9. doi: $10.1126 /$ science.276.5319.1665
34. Greenfield NJ, Using circular dichroism spectra to estimate protein secondary structure. Nat Protoc (2006) 1:2876-90. doi: 10.1038/nprot.2006.202

35. Ramsay GD, Eftnik MR. Analysis of multidimensional spectroscopic data to monitor unfolding of proteins. Methods Enzymol. (1994) 240:615-45. doi: 10.1016/S0076-6879(94)40066-0

36. Otwinowski Z, Minor W. Processing of X-ray diffraction data collected in oscillation mode. Methods Enzymol. (1997) 276:307-26. doi: 10.1016/S0076-6879(97)76066-X

37. McCoy AJ. Solving structures of protein complexes by molecular replacement with Phaser. Acta Cryst. (2007) 63:32-41. doi: 10.1107/S09074449060 45975

38. Adams PD, Afonine PV, Bunkoczi G, Chen VB, Davis IW, Echols $\mathrm{N}$, et al. PHENIX: a comprehensive Python-based system for macromolecular structure solution. Acta Cryst. (2010) 66:213-221. doi: 10.1107/S0907444909052925

39. Emsley P, Cowtan K. Coot: model-building tools for molecular graphics. Acta Cryst. (2004) 60:2126-32. doi: 10.1107/S09074449040 19158

40. Davis IW, Leaver-Fay A, Chen VB, Block JN, Kapral GJ, Wang $\mathrm{X}$, et al. MolProbity: all-atom contacts and structure validation for proteins and nucleic acids. Nucleic acids research. (2007) 35:W375-83. doi: $10.1093 / \mathrm{nar} / \mathrm{gkm} 216$

Conflict of Interest: The authors declare that the research was conducted in the absence of any commercial or financial relationships that could be construed as a potential conflict of interest.

Copyright () 2020 Zhou, Zaidi, Ton and Fera. This is an open-access article distributed under the terms of the Creative Commons Attribution License (CC BY). The use, distribution or reproduction in other forums is permitted, provided the original author(s) and the copyright owner(s) are credited and that the original publication in this journal is cited, in accordance with accepted academic practice. No use, distribution or reproduction is permitted which does not comply with these terms. 